\title{
Assessment of ICAM-1 gene polymorphism in Acne Vulgaris
}

\author{
A.A.Ibrahim, G.M.Abdel-khalik and M.H.Hassanein
}

Dermatology, Venerology and Andrology, Dept., Faculty of Medicine, Benha Univ., Benha, Egypt E-Mail:

\section{Abstract}

Background: Considering the recognized roles of ICAM-1 in inflammation and rising an evidence that inflammation is evident at all stages of AV. The current study aimed to investigate the incidence of ICAM-1 gene expression and polymorphism with special reference to amino acids at position 469 and 241 with acne vulgaris and its possible correlation with the lesion severity. Patient and methods: The present case control study included 60 participants of matched age, sex and of Egyptian origin where there were 45 acne patients assigned into three groups according to acne severity (mild, moderate and severe), 15 patients in each group, and there were 15 participants as control.All studied participants were tested for the genotypes of ICAM-1 gene polymorphism by PCR where four primers have been used to detect all possible polymorphism at codon 241 and 469 Results: There was statistically significant difference among four studied groups as regard ICAM (496) and ICAM (241) polymorphism where the highest percentage of GG were among severe acne group and none of controls had GG genotype. There was significant association between ICAM (496) polymorphism and patients' BMI where the highest BMI Conclusion: ICAM-1 496 and 241 polymorphism might be associated with AV. Further understanding of functional significance of the ICAM-1 polymorphism will help delineate immunopathogenesis in certain subgroups of AV obese patients

Keywords: ICAM-1 gene, Acne Vulgaris, GAGS grading.

\section{Introduction}

Acne vulgaris (AV) is an extremely common condition with a lifetime prevalence of approximately $85 \%$ and occurs mostly during adolescence across the globe [1].

Four key pathogenic processes associated with the formation of acne lesions, include: 1) Alteration of follicular keratinization that leads to comedones. 2) Increased and altered sebum production under androgen control. 3) Bacterial colonization mainly by Propionibacterium acnes, and 4) Complex inflammatory mechanisms that involve both innate and acquired immunity [2].

The formation of the micro-comedone is preceded by a mononuclear infiltrate formed mainly by CD4+ T lymphocytes and CD68+ macrophages, that explain the inflammatory process in the early stages of acne (3).

In some mechanistic studies it has been found that IL1, intercellular adhesion molecule 1 (ICAM-1), tumor necrosis factor alpha (TNF- $\alpha$ ) induce differentiation of human sebocytes into keratinocytes like phenotype, resulting in hyperkeratosis in the infundibulum in acne vulgaris [4].

ICAM-1 over expressed by endothelial cells during inflammation, resulting in increased firm leukocyteendothelial cell adhesion and transmigration of leukocytes at sites of inflammation. Therefore, the loss of ICAM-1 inhibits wound healing, keratinocyte migration from the edges of the wound toward the center, and granulation tissue formation [5]. ICAM1 not only expressed on vascular endothelium but also in antigen presenting cells, and activated lymphocytes [5].

The polymorphisms having been reported for ICAM1 in exons 4, and 6. The polymorphisms at these positions 241 and 469 can alter ligand binding or the stability of the multimeric ICAM-1 on the cell surface, and therefore alter signal transduction [6].
Significant association was found between the presence of skin lesions and the studied polymorphism in the distribution of the K469E allele [8] the same reported with R241 allel [8].

Despite the known correlation between I-CAM-1 and skin lesion. However, little is known about the prevalence of different polymorphism and its correlation with acne severity

The aim of this work was to investigate the incidence of ICAM-1 gene expression and polymorphism with special reference to amino acids at position 469 and 241 with acne vulgaris and its possible correlation with the lesion severity.

\section{Patient and Method}

This case- control study was conducted on a total number of 60 subjects from the outpatient clinic of Dermatology, Venereology and Andrology Department of Benha University Hospitals from July to December 2020, 45 patients suffering from Acne Vulgaris (AV) (Group A) and 15 apparently healthy individuals of matched age and sex as a control group (Group B).

The study was approved by the local ethic committee of Benha Faculty of Medicine. Informed consent was obtained from each individual before sample collection.Subjects with any of the following conditions were excluded from the study:

- Acniform eruption, acne conglobate and fulminating acne.

- Inflammatory or autoimmune cutaneous or systemic diseases.

- Malignancy.

- Chronic systemic diseases eg:diabetes mellitus,liver or kidney or cardiac diseases.

All patients were subjected to Full history taking, complete general examination. Complete cutaneous 
examination and Grading of Acne Vulgaris using the Global Acne Grading System (GAGS)

All studied subjects were tested for the genotypes of I CAM1 gene polymorphism by Polymerase Chain Reaction (PCR) and ICAM gene expression by quantitative reverse transcription PCR (RT-qPCR).

\subsection{Statistical analysis}

The results have been tabulated and statistically analyzed using software package SPSS. Only $\mathrm{P}<0.05$ considered as significant. The collected data was revised, coded, tabulated, and introduced to a PC using Statistical package for Social Science (IBM Corp. Released 2011. IBM SPSS Statistics for Windows, Version 20.0. Armonk, NY: IBM Corp.). Data were presented and suitable analysis was done according to the type of data obtained for each parameter.

\section{Results}

There were no statistically significant differences among four studied groups regarding age /years and sex. Most mild acne patients were males $<20$ years while severe acne was more presentable among female aged from 21 years to 25 years .

There was statistically significant difference among studied groups as regard BMI where severe acne group had the highest BMI $(26.73 \pm 3.57 \mathrm{Kg} / \mathrm{m})$

There was statistically significant difference among three acne-infected groups regarding affected sites where $93.3 \%$ of mild acne group were face only affected while face, shoulder and back were affected in $26.7 \%$ of severe acne group. Also, there was statistically significant difference among three acne-infected groups regarding received treatment where all patient $\mathrm{s}$ in severe acne group received both topical and systemic treatment.

Face only was the most affected site in all acne patients while affection of face, shoulders and back was encountered among severe acne patients only .

There was statistically significant difference among four studied groups as regard ICAM (496) polymorphism where the highest percentage of GG $(55.6 \%)$ were among severe acne group and none of controls had GG genotype

the highest percentage of AA genotype (36.4\%) were among control group while the highest percentage of AG and GG genotypes were among severe acne group $(38.9 \% \& 55.6 \%$ respectively) and none of controls had GG genotype .

That there was statistically significant difference among four studied groups as regard ICAM (241) polymorphism where the highest percentage of GG $(60 \%)$ were among severe acne group and none of controls had GG genotype.

the highest percentage of AA genotype (46.2\%) were among control group while the highest percentage of AG was among moderate acne patients (37.5\%) and GG genotype were the highest among severe acne group $(60 \%)$ and none of controls had GG genotype .

There was significant association between ICAM (496) polymorphism and patients' BMI where the highest BMI $(28 \pm 2 \mathrm{Kg} / \mathrm{m})$ was among acne patients with GG genotype .

There was highly statistically significant association between ICAM (241) polymorphism and patients' BMI where the highest BMI $(28.7 \pm 2 \mathrm{Kg} / \mathrm{m})$ was among acne patients with GG genotype.

This figure shows that in acne patients GG genotype of both ICAM 496 and ICAM 241 was associated with higher BMI .

There was highly statistically significant difference among studied groups as regard gene expression where severe acne group had the highest gene expression (2.07 \pm 0.6$)$.

There was highly statistically significant association between ICAM (496) polymorphism and gene expression in acne patients where the highest gene expression (2.4 \pm 0.4$)$ was among acne patients with GG genotype.

This table shows that there was highly statistically significant association between ICAM (241) polymorphism and gene expression in acne patients where the highest gene expression $(2.3 \pm 0.42)$ was among acne patients with GG genotype.
AA

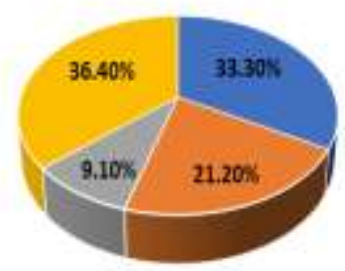

- Mild acre group $\quad$ "Moderate ache group
- Severe acne group $\quad$ icontrol group
AG

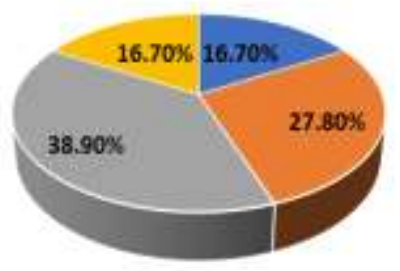

- Mild acne group
= Severe acne group

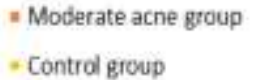

- Contral group

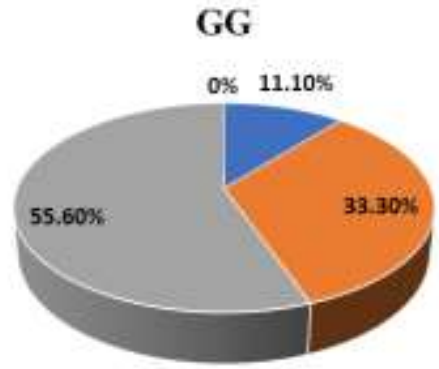

- Mild acre group

- Sever acne group
" Moderate acne group

- Contrd group

Fig. (1) Pie diagram shows comparison of ICAM (496) polymorphism among the four studied groups. 


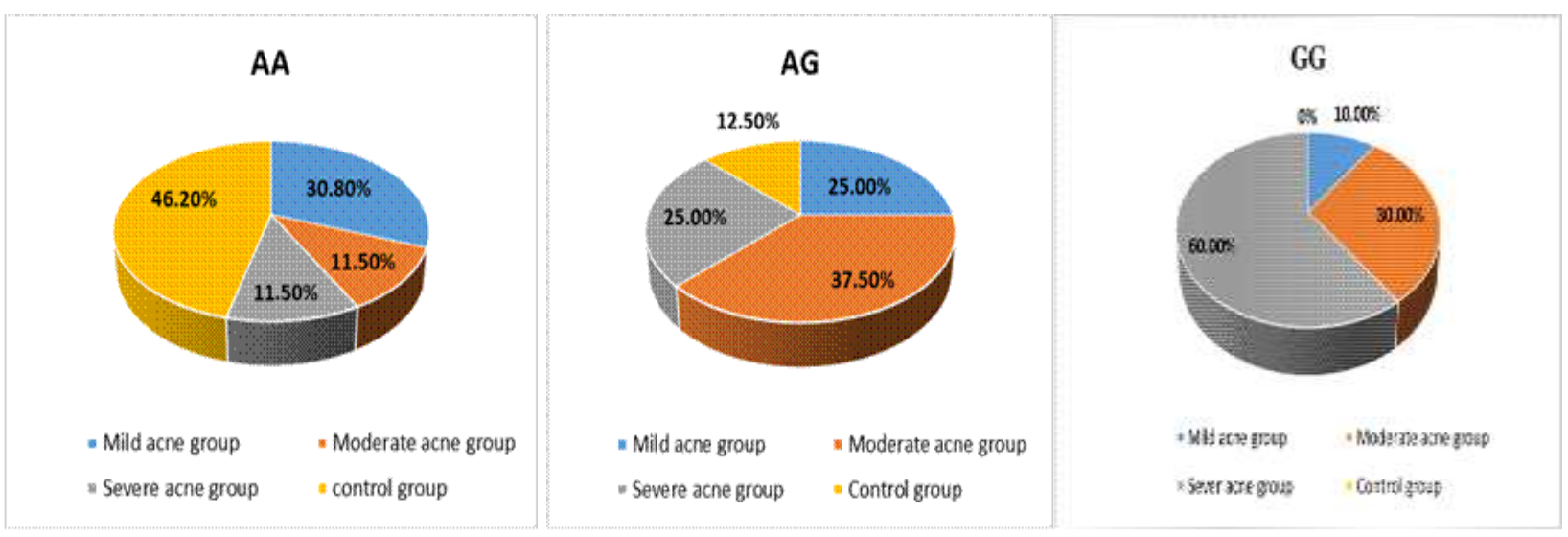

Fig. (2) Pie diagram shows comparison of ICAM (241) polymorphism among the four studied groups.

\section{Discussion}

ICAM-1 has been shown to be important in the pathogenesis and outcome of many inflammatory diseases. For example it is implicated as genetic risk factor in type 1/type 2 diabetes, Graves' disease, multiple sclerosis, inflammatory bowel diseases and Behçet's disease [7].

In the present study, there were significant associations between ICAM (241\&496) polymorphism and acne patients' BMI where the highest BMI was among acne patients with GG genotype that was highly expresses in acne patients. This indicates that there is association between obesity and ICAM genotype expression as obesity enhances expression of ICAM-1.

In accordance with a study, Lin et al., [9] aimed to investigate the effect of visfatin, that is known to act as a mediator in obesity, on the adhesion of THP-1 monocytes to human vascular endothelial cells through measurement of ICAM-1 and VCAM-1 expression in endothelial cells by western blotting concluded that visfatin promoted monocyte-endothelial cell adhesion by increasing ICAM-1 and VCAM-1 expression via the activation of $\mathrm{p} 38 / \mathrm{PI} 3 \mathrm{~K} / \mathrm{Akt}$ signaling and downstream ROS production and IKK/NF-кB activation. This indicates that obesity promotes ICAM-1 expression and consequently according to our results increase incidence of severity of AV.

Regarding ICAM-1 gene polymorphism and its expression, it was found that that there were statistically significant differences among the studied groups as regard ICAM (241\&496) polymorphism where the highest percentage of GG were among severe acne group and none of controls had GG genotype. There was highly statistically significant difference among studied groups as regard gene expression where severe acne group had the highest gene expression $(2.07 \pm 0.6)$. There were highly statistically significant association between ICAM (241\&496) polymorphism and gene expression in acne patients where the highest gene expression was among acne patients with GG genotype. This indicates that ICAM-1 (469 and 241) gene polymorphism and expression are prevalent in acne patients and it's mostly correlated with the AV severity.
These results were supported by Su et al. [10] who showed among his study results that the expression of TLR-2, NF- $\mathrm{KB}$ and the expression of its down-stream factor ICAM-1 increased in the inflammatory skin tissue induced by $\mathrm{P}$. acnes.

In accordance with Pretsch et al., [11] study which included treatment of $\mathrm{AV}$ with $\mathrm{T}$. wortmannii compounds demonstrated that the most effective therapeutic agents to improve acne disease which had prominently down-regulate ICAM-1 expression in endothelial cells after TNF- $\alpha$ stimulation and IL-8 secretion in keratinocytes after P. acnes treatment via the $\mathrm{NF}-\mathrm{kB}$ and MAPK pathways .

Expression of ICAM-1 is associated with the degree and nature of the inflammation. Increased expression of ICAM-1 may induce leukocyte and keratinocyte interaction and is considered an important initiator in numerous types of inflammatory skin diseases [12].

Regarding a study aims to investigate the pattern of expression of adhesion molecules in evolving acne lesions included forty-nine patients with moderate to severe acne classified according to their duration of inflammation as up to $6 \mathrm{~h}$, from 6 to $24 \mathrm{~h}$, from 24 to 48 $\mathrm{h}$ and from 48 to $72 \mathrm{~h}$. and were biopsied following lesion mapping found that ICAM-1 was universally expressed by basal keratinocytes, inflammatory cells and cells in the interstitial dermis of acne lesions of $6 \mathrm{~h}$ duration and expression was consistent up to $72 \mathrm{~h}$. ICAM-1 is expressed constitutively by endothelial cells and up-regulated endothelial cell expression of ICAM-1 has been shown to be a feature of both specific and nonspecific cutaneous inflammation. Keratinocyte expression of ICAM-1 has, however, been reported to be minimal in normal skin but induced by TNF-a and IFN-g and not IL-1 [13].

ICAM-1 is an important adhesion molecule that mediates the adhesion reaction and promotes the adhesion of inflammatory sites, and studies have confirmed that it is regulated by NF-KB ICAM-1 is an important adhesion molecule that mediates the adhesion reaction and promotes the adhesion of inflammatory sites, and studies have confirmed that it is regulated by $\mathrm{NF}-\mathrm{\kappa B}$ 
We found that the TLR-2/NF- $\mathrm{BB}$ signaling pathway and its downstream factor ICAM-1 were activated in $\mathrm{HaCaT}$ cells infected with $\mathrm{P}$. acnes, we investigated the effects of LF on the generation of inflammatory cytokines in $\mathrm{HaCaT}$ cells induced by heat-killed $\mathrm{P}$.

The SNP rs7531806 is located at the loci 1q24.2, covering a gene cluster including SELL (selectin L), SELP (selectin P) and SELE (selectin E). These selectins of adhesion molecules have important roles in regulating homoeostasis and cutaneous inflammation. In mice lacking ICAM-1 expression, the healing of wounds is delayed, probably due to the decreased leukocyte accumulation into the wound site (14).

Finally, the correlation between ICAM-1 mRNA gene expression and inflammation is well defined in several study that agree with our conclusion where AV pathogenesis is based on inflammatory reaction.

It was concluded that ICAM-1 mRNA gene expression is statistically significant associated with moderate and severe acne patient and more evident among those had GG genotype that was more prevalent among severe acne group.

Further research is needed to define the correlation between severity and ICAM-1gene expression via invitro dose dependent experiments.

\section{Conclusion}

ICAM-1 496 and 241polymorphism might be associated with AV. Further understanding of functional significance of the ICAM-1 polymorphism will help delineate immunopathogenesis in certain subgroups of AV obese patients

\section{References}

[1] D.Lynn, T.Umari, A.Dunnick and P.Dellavalle. The epidemiology of acne vulgaris in late adolescence. Adolesc Health Med Ther.vol. 7,pp. 13-25,2016.

[2] A.Tan, B.Schlosser and A.Paller. A review of diagnosis and treatment of acne in adult female patients. Inter $\mathrm{j}$ of wom dermatol.vol.4(2),pp. 5671,2018 .

[3] S.Das and R V. Reynolds. Recent advances in acne pathogenesis: implications for therapy. Am J Clinical Dermatol.vol.15(6),pp. 479-488,2014.

[4] B.Holland, H.Jeremy, G.Roberts, C.Seukeran and J.Cunliffe. Inflammation in acne scarring: a comparison of the responses in lesions from patients prone and not prone to scar. $\mathrm{Br} \mathrm{J}$ Dermatol.vol. 150(1),pp. 72-81,2004.

[5] T.Nagaoka, Y.Kaburagi, Y.Hamaguchi, M.Hasegawa, K.Takehara, DA.Steebe. Delayed wound healing in the absence of intercellular adhesion molecule-1 or L-selectin expression. The Am j of pathol.vol.157(1),pp. 237-247,2000.

[6] H.Harjunpää, M.Llort Asens, C.Guenther and C.Fagerholm. Cell Adhesion Molecules and Their Roles and Regulation in the Immune and Tumor Microenvironment. Front in immunol.vol. 10,pp. 1078-1078,2019.

[7] I.Ben Dhifallah, EF. Karray, F.Sassi and K.Hamzaoui. Intercellular adhesion molecule 1 $\mathrm{K} 469 \mathrm{E}$ gene polymorphism is associated with presence of skin lesions in Tunisian Behcet's disease patients. Tissue Antigens.vol. 75(1),pp. 7478,2010 .

[8] L.Boiardi, C.Salvarani, B.Casali, I.Olivieri, G.Ciancio, F.Cantini. Intercellular adhesion molecule-1 gene polymorphisms in Behcet's Disease. J of Rheumatol.vol.28(6), pp.1283-1287. ,2001.

[9] T.Lin, K.Chen, Y.Jian, C.Hsu, C.Huang, T.Kuan, and C.Juan. Visfatin Promotes Monocyte Adhesion by Upregulating ICAM-1 and VCAM-1 Expression in Endothelial Cells via Activation of p38-PI3KAkt Signaling and Subsequent ROS Production and IKK/NF- $\kappa B$ Activation. international journal of experimental cellular physiology, biochemistry, and pharmacol.vol. 52(6),pp. 1398-1411,2019.

[10] Y.Su, W.Cui, and H.Wei. Influence oflactoferrin on Propionibacterium acnes-induced inflammation invitro and in vivo. Dermatol Therapy.vol. 33,pp.e14483,2020.

[11] A.Pretsch, M.Nagl, K.Schwendinger, B.Kreiseder, M.Wiederstein. Antimicrobial and Anti-Inflammatory Activities of Endophytic Fungi Talaromyces wortmannii Extracts against AcneInducing Bacteria. PLoS ONE.vol. 9(6),pp. e97929,2014.

[12] H.Huang, C.Chang, S.Hu, Y. Hsiao and J. Wu. Spilanthol inhibits TNF- $\alpha$-induced ICAM-1 expression and pro-inflammatory responses by inducing heme oxygenase-1 expression and suppressing pJNK in HaCaT keratinocytes. Mol Med Rep.vol. 18(3),pp. 2987-2994,2018.

[13] M. Layton, C.Morris, J.Cunliffe, E.Ingham. Immunohistochemical investigation of evolving inflammation in lesions of acne vulgaris. Exp Dermatol.vol. (7),pp. 191-197,1998.

[14] L.He, WJ.Wu, JK. Yang. Two new susceptibility loci 1q24.2 and $11 \mathrm{p} 11.2$ confer risk to severe acne. Nat Commun.vol. 5,pp. 2870 ,2014. 